\section{Characterizing the activation of the Wnt signaling pathway in hilar cholangiocarcinoma using a tissue microarray approach}

\author{
W. Chen, ${ }^{1}$ L. Huang, ${ }^{1}$ J. Liang, ${ }^{2}$ J. Cai, ${ }^{1}$ \\ Y. Lei, ${ }^{2}$ J. Lai, ${ }^{1}$ L. Liang, ${ }^{1}$ K. Zhang' \\ 'Department of Pancreatobiliary Surgery, \\ The First Affiliated Hospital, Sun Yat-sen \\ University, Guangzhou, China \\ 2Department of Medical Ultrasonics, The \\ First Affiliated Hospital, Institute of \\ Diagnostic and Interventional Ultrasound, \\ Sun Yat-sen University, Guangzhou, \\ China
}

\section{Abstract}

Hilar cholangiocarcinoma (HCCA) is an invasive hepatic malignancy that is difficult to biopsy; therefore, novel markers of HCCA prognosis are needed. Here, the level of canonical Wnt activation in patients with HCCA, intrahepatic cholangiocarcinoma (IHCC), and congenital choledochal cysts (CCC) was compared to understand the role of Wnt signaling in HCCA. Pathology specimens from HCCA $(\mathrm{n}=129)$, IHCC $(\mathrm{n}=31)$, and CCC $(\mathrm{n}=45)$ patients were used to construct tissue microarrays. Wnt2, Wnt3, $\beta$-catenin, TCF4, c-Myc, and cyclin D1 were detected by immunohistochemistry. Parallel correlation analysis was used to analyze differences in protein levels between the HCCA, IHCC, and CCC groups. Univariate and multivariate analyses were used to determine independent predictors of successful resection and prognosis in the HCCA group. The protein levels of Wnt2, $\beta$-catenin, TCF4, $\mathrm{c}$ Myc, and cyclin D1 were significantly higher in HCCA compared to IHHC or CCC. Wnt signaling activation (Wnt2+, Wnt3+, nuclear $\beta$ catenin+, nuclear TCF4+) was significantly greater in HCCA tissues than CCC tissues. Univariable analyses indicated that expression of cyclin D1 as well as Wnt signaling activation, and partial Wnt activation (Wnt2+ or Wnt3+ and nuclear $\beta$-catenin+ or nuclear TCF4+) predicted successful resection, but only cyclin D1 expression remained significant in multivariable analyses. Only partial Wnt activation was an independent predictor of survival time. Proteins in the canonical Wnt signaling pathway were present at higher levels in HCCA and correlated with tumor resecility and patient prognosis. These results suggest that Wnt pathway analysis may be a useful marker for clinical outcome in HCCA.

\section{Introduction}

Cholangiocarcinoma (CCA) is a highly invasive, malignant tumor that occurs along the biliary duct. ${ }^{1}$ CCA is classified based on its localization as: i) intrahepatic cholangiocarcinoma (IHCC); ii) extrahepatic cholangiocarcinoma (EHCC); or iii) hilar (or perihilar) cholangiocarcinoma (HCCA). IHCC is an aggressive primary tumor originating in the epithelial cells of the intrahepatic bile ducts, estimates of disease free survival after 5 -years range from $2-41 \%{ }^{2}$ HCCA originates from cells in the hilar biliary duct epithelium, is highly metastatic, and is the most common malignant tumor in the biliary system, ${ }^{3}$ accounting for $50 \%-60 \%$ of all CCA. HCCA is often diagnosed late, and previous studies have shown that after accounting for other variables, the histological grade of the tumor and microscopically negative tumor margins were significantly associated with overall survival following surgical resection for HCCA. ${ }^{4,5}$ However, other studies have shown no association between tumor stage and prognosis. ${ }^{6}$ Therefore, identifying novel prognostic markers for HCCA would have clinical utility.

In contrast to $\mathrm{CCA}$, congenital choledochal cysts (CCC), are relatively benign and have a minimal risk of developing cancer. CCC refers to the congenital dilatation of intra- or extrabile duct $^{7}$ due to cyst formation. CCC mostly occurs in the common bile duct. Choledochal cysts are treated by surgical excision. ${ }^{8}$ Complications resulting from these cysts include cholangitis and a $2 \%$ risk of malignant transformation.

The canonical Wnt pathway is involved in many cancers ${ }^{9,10}$ and has been implicated in the development of HCCA. C-myc is a major downstream target gene in the canonical Wnt pathway. ${ }^{11,12}$ Previous work has also shown that $\beta$-catenin, a second Wnt target, can promote cholangiocarcinoma growth via interactions with GSK3 $\beta .{ }^{13}$ However, it is not clear whether aberrant activation of the Wnt pathway occurs in cases of CCA, particularly HCCA. Aberrant methylation of Wnt pathway genes has been reported in IHCC leading to $\beta$-catenin stabilization and abnormally high levels of Wnt signaling. ${ }^{14}$ We have previously reported that in vitro IHCC and HCCA cell lines express high levels of Wnt2, Wnt3, TCF4, $\beta$-catenin, c-myc, and cyclin D1, and that canonical Wnt signaling is activated in both IHCC and HCCA cell lines. ${ }^{15}$ Knockdown of Wnt2 and $\beta$-catenin increased apoptosis and reduced proliferation in an HCCA cell line in vitro, suggesting the Wnt pathway may be important for tumor genesis and could be used as a prognostic marker. ${ }^{15}$ It remains unclear whether the Wnt pathway is activated during development of HCCA
Correspondence: Dr. Kunsong Zhang, Department of Pancreatobiliary Surgery, The First Affiliated Hospital of Sun Yat-sen University, 58 Zhongshan Road 2, Guangzhou 510080, Guangdong, China.

Tel. +86.20.87755766-8214 - Fax +86.20.87755766-8663. E-mail: zhangkunsong@263.net

Key words: Hilar cholangiocarcinoma; Wnt signaling pathway; tissue microarray; $\beta$-catenin; c-Myc; cyclin D1.

Contributions: WC, writing of the manuscript first draft; WC, LH, performed research/study; LL, contributed important reagents; LY, JLai, literature searches and analyses; JLiang, JC, statistical analysis; KZ, study design, protocol writing. All the listed authors have participated actively in the study and all meet the requirements of the authorship.

Conflict of interest: the authors declare no conflict of interest.

Funding: this work was supported by the Medical Scientific Research Foundation of Guangdong Province (A2012088, B2013106) and the Guangdong Natural Science Foundation (S2012040006299).

Received for publication: 18 May 2015.

Accepted for publication: 28 December 2015.

This work is licensed under a Creative Commons Attribution-NonCommercial 4.0 International License (CC BY-NC 4.0).

(C) Copyright W. Chen et al., 2016

Licensee PAGEPress, Italy

European Journal of Histochemistry 2016; 60:2536 doi:10.4081/ejh.2016.2536

and whether Wnt activation separates benign, malignant, and highly malignant lesions. The primary objective of this study was to determine whether differences in Wnt associated protein levels or activation of the Wnt signaling pathway were associated with the potential for malignancy in HCCA (highly malignant with metastatic potential), IHCC (malignant with metastatic potential), ${ }^{16}$ and CCC (precancerous or benign cancerous with minimal risk of tumorigenesis) ${ }^{7}$ lesions. We also considered whether the progression from cyst to cancerous lesion to hilar cancer was inevitable.

\section{Materials and Methods}

\section{Patient cohorts}

The study was conducted in accordance with the ethical guidelines of the Declaration of Helsinki and approved by the Institutional Review Board of The First Affiliated Hospital, 
Sun Yat-sen University. The Institutional Review Board approved the retrospective analysis of anonymous data (IRB approval no. [2012]056).

Resected tissue samples were obtained from patients with HCCA $(n=129)$, IHCC $(n=31)$, or CCC $(n=45)$ seen at the First Affiliated Hospital of Sun Yat-sen University (Guangzhou, China). All of the diagnoses were confirmed by the pathologist at the hospital. Of the HCCA patients, 102 were confirmed adenomas and 36 were unspecified tumors. The tumors were graded as well differentiated $(n=24)$, moderately differentiated $(n=40)$, or poorly differentiated $(n=10)$ based on the Union for International Cancer Control (UICC) standard definitions. The grade was not determined for the remaining $(\mathrm{n}=19)$ tumors. The resected tissue was collected from July 1998May 2007. The average age of the HCCA patients was 55.9 years (range: $20-82$ ) and the majority of the HCCA cases were male (91/102; $70.5 \%)$. The resected tissue from IHCC patients was collected from July 2006-August 2008. The average age of the IHCC patients was 57.3 years (range: $28-82$ ), $61.3 \%$ of IHCC patients were male. The resected tissue from CCC patients was collected from March 2005August 2008. The average age of the CCC patients was 30.1 years (range: 1-59) and $28.9 \%$ were male.

\section{Preparation of the tissue microarrays}

To make the tissue microarrays (TMA), recipient paraffin blocks (97.5 g Leica paraffin, $2.4 \mathrm{~g}$ beeswax) were arranged in $10 \times 8 \mathrm{~mm}$ or 11 x $9 \mathrm{~mm}$ microarrays. The empty chambers were $1.0 \mathrm{~mm}$ in diameter, $3.0 \mathrm{~mm}$ in depth, and separated by $0.75 \mathrm{~mm}$. Needles $(1.0 \mathrm{~mm}$ diameter) were used to remove cores from the tissue blocks containing the resected tissue specimens ( $n=3 /$ specimen), which were placed in the recipient paraffin blocks. The TMA blocks were heated for $1 \mathrm{~h}$ in a $42^{\circ} \mathrm{C}$ oven and cooled to room. Next, the wax blocks were immersed in liquid wax for 1-3 $s$ before the surface of the block was flattened to preserve the antigenicity. For sectioning, the TMA blocks were precooled at $4^{\circ} \mathrm{C}$ for $4 \mathrm{~h}$ and $15^{\circ} \mathrm{C}$ for $15 \mathrm{~min}$. The blocks were then quickly mounted on a microtome and serially sectioned in 2-5 m thick sections, 20-30 sections were cut per block. Serial sections were transferred to slides, dried at room temperature, and then put on a slide dryer plate at $62^{\circ} \mathrm{C}$ for 3 min to melt the wax and expose the tissue specimens. The sections were dried at $58^{\circ} \mathrm{C}$ for an additional $18 \mathrm{~h}$ and stored at $-20^{\circ} \mathrm{C}$ until staining.

\section{Reagents}

Rabbit anti-human and goat anti-human primary antibodies against Wnt2, Wnt3, TCF4, c-Myc, cyclin D1, and $\beta$-catenin (Santa Cruz
Biotechnology Inc., Santa Cruz, CA, USA) were used at a 1:100 dilution. The SP test kits, ready-to-use $2^{\text {nd }}$ generation $\mathrm{IHC}$ Eli vision ${ }^{\mathrm{TM}}$ plus kit was used for DAB (3,3-diaminobenzidine) visualization, horseradish peroxidase labeled anti-mouse and anti-rabbit antibodies, and a 5\% BSA blocking solution were purchased from Key GENE Biotech (Nanking, China).

\section{Immunohistochemistry}

Sections were incubated at $70^{\circ} \mathrm{C}$ for $1 \mathrm{~h}$, then de-paraffinized in dimethylbenzene and rehydrated in graded ethanol solutions (100, 90 , and $75 \%$ ). For antigen retrieval, slides were treated for 15-20 min with $10 \mathrm{mM}$ sodium citrate buffer $(\mathrm{pH} 6.0)$ at $92-98^{\circ} \mathrm{C}$. The slides were allowed to cool in the buffer to room temperature and incubated with $0.3 \% \mathrm{H}_{2} \mathrm{O}_{2}$ for 10 min to block endogenous peroxidase activity. The slides were then washed with phosphatebuffered saline (PBS) and incubated in primary antibody for $2 \mathrm{~h}$ at room temperature. After washing with PBS, sections were incubated with Reagent A of the Eli vision ${ }^{\mathrm{TM}}$ plus kit at room temperature for $20 \mathrm{~min}$ and washed again with PBS. Then, Eli vision ${ }^{\mathrm{TM}}$ plus kit Reagent B: Poly-HRP-Anti Mouse/Rabbit IgG was added and the slides were incubated for 30 min. After another PBS wash, the peroxidase reaction was developed using a freshly prepared 3, 3'-diaminobenzidine solutions. Tap water was added to stop the reaction. H\&E stained sections from the same tissue blocks were used to confirm the pathological characteristics of each sample. For long-term storage of the slides, sections were stained with hematoxylin for $10-15 \mathrm{~s}$, differentiated in $1 \%$ hydrochloric acid alcohol for 1-2 s, treated with bluing in running water for $10 \mathrm{~min}$, dehydrated by gradient ethanol treatments, and incubated with dimethylbenzene for $3 \mathrm{~min}$. The sections were then sealed with neutral glue and allowed to dry. Negative control staining was performed substituting saline buffer for the primary antibodies.

\section{Criteria for evaluating the IHC staining results}

Tissue samples were considered positive for Wnt2, Wnt3, and c-Myc protein expression if brown-yellowish granules were seen in the cytoplasm or plasma membrane. For cyclin D1, tissue samples were only considered positive if staining was apparent in the nucleus. For TCF4 and $\beta$-catenin, cytoplasmic expression was defined as brown-yellowish staining in the cytoplasm or plasma membrane; while staining in the nucleus was considered positive nuclear protein.

The abundance of each marker was scored (+/-) by two blinded, independent expert pathologists. Representative areas $(n=5)$ from each slice were selected based on the staining region and intensity. One hundred tumor cells per field were observed under $400 \times$ magnifica-

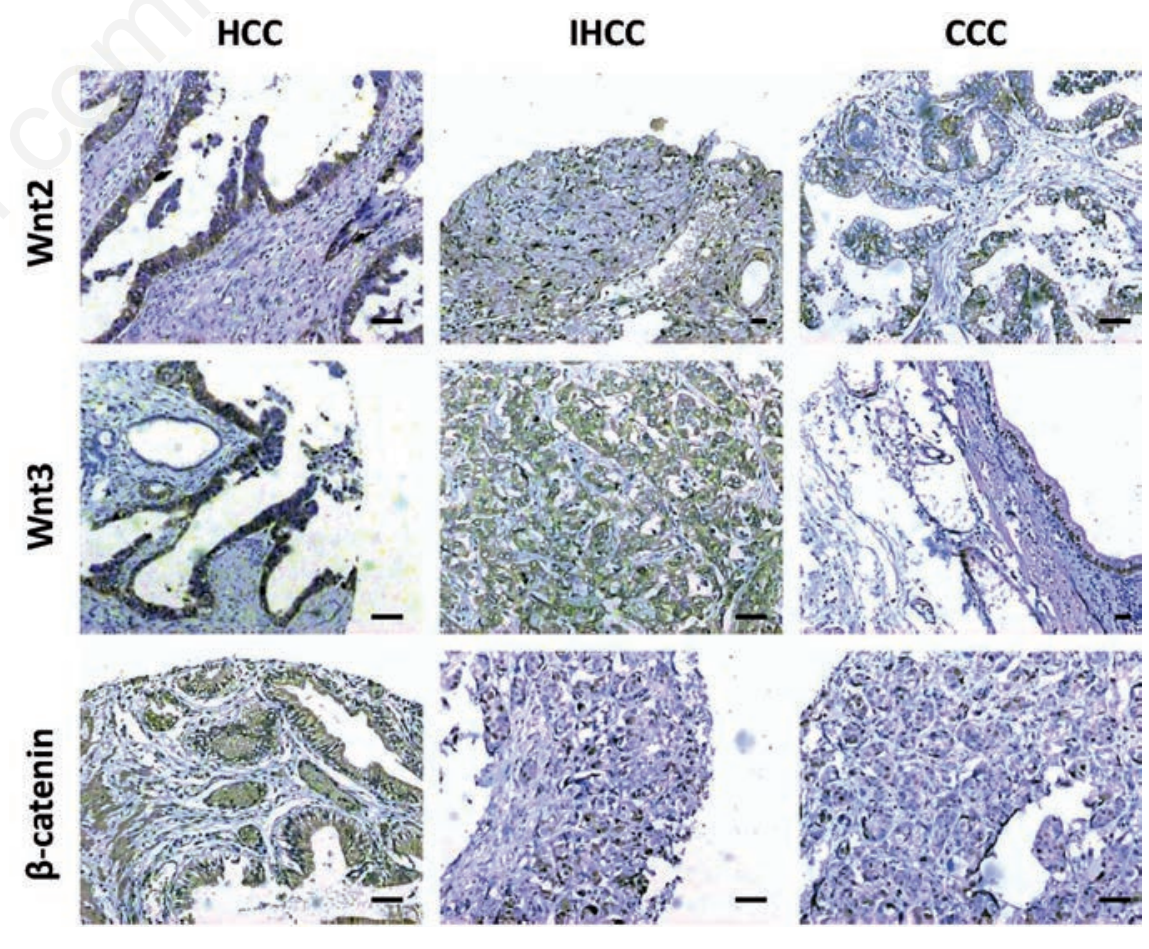

Figure 1. Representative immunohistochemistry images of Wnt2, Wnt3 and $\beta$-catenin proteins (upper, middle and lower panel, respectively). Scale bars: $100 \mu \mathrm{m}$. 
tion to determine the number of positive cells. The protein level for each marker was scored using the following scale: -, none; +, less than $10 \%$ positively stained cells;,$++ 10 \%-49 \%$ positively stained cells; +++ , more than $50 \%$ positively stained cells. The number of tumor cells counted $(n=100)$ was used as the denominator for determining the percent positive.

The activity of the Wnt pathway for each tissue sample was based on positive staining $(+$, ++ , or +++ ) for the individual markers Wnt2, Wnt3, nuclear $\beta$-catenin, and nuclear TCF4 in serial sections. Wnt pathway activation was defined as Wnt2+, Wnt3+, nuclear $\beta$-catenin+, and nuclear TCF4+; and partial Wnt activation was defined as Wnt $2+$ or Wnt $3+$ and $\beta$ catenin+ or TCF4+.

\section{Statistical analyses}

SPSS ver. 13.0 (IBM, New York, NY, USA) was used for the analyses. Survival was assessed using the Kaplan-Meier analysis, and a Log Rank test was used to assess differences between groups. Multivariate regression analysis was used to model the Cox proportional hazard ratio. The relationship between factors was analyzed using Spearman correlations. A $\mathrm{P}<0.05$ was considered statistically significant.

\section{Results}

\section{Expression levels of Wnt pathway- related proteins}

We first determined if the levels of the Wntassociated signaling markers (Wnt2, Wnt3, TCF4, $\beta$-catenin, c-myc, and cyclin D1) were correlated in HCCA tissues. Wnt2 levels positively correlated with c-myc $(\mathrm{r}=0.196$, $\mathrm{P}=0.031$ ), but negatively correlated with partial Wnt pathway activation ( $\mathrm{r}=-0.195$, $\mathrm{P}=0.030$ ). Wnt3 levels positively correlated with cytoplasmic $\beta$-catenin levels $(\mathrm{r}=0.223$, $\mathrm{P}=0.012$ ). Wht pathway activation positively correlated with both $\beta$-catenin $(\mathrm{r}=0.730$, $\mathrm{P}<0.01)$ and nuclear TCF4 staining $(\mathrm{r}=0.311$, $\mathrm{P}<0.01)$. Potential Wnt pathway activation was positively correlated with confirmed Wnt pathway activation $(\mathrm{r}=0.229, \mathrm{P}<0.01)$.

To determine whether the expression of Wnt proteins differed between malignancies, we compared the levels of Wnt pathway related proteins between HCCA, IHCC, and CCC samples. The immunohistochemistry images of Wnt2 protein were shown in Figure 1, upper panel. The levels of Wnt2 were significantly higher in the HCCA (100\%) samples compared to the IHCC (93.6\%) and CCC (86.7\%) samples $(\mathrm{P}<0.001$; Figure $2 \mathrm{~A})$. The expression of Wnt2 was not significantly different between the IHCC and CCC groups $(\mathrm{P}=0.214)$. Strongly pos- itive cells $(++$ or +++$)$ were more frequent in samples from the HCCA group (99.1\%) than the IHCC (54.9\%) and CCC (71.1\%) groups, but the difference was not statistically significant. The immunohistochemistry images of Wnt3 protein were shown in Figure 1, middle panel. Wnt3 was present at similar levels in the HCCA (93.0\%), IHCC (93.6\%), and CCC (95.6\%) samples (Figure 2B). Thus, the level of Wnt2, but not Wnt3, distinguished HCCA from IHCC and CCC.

\section{$\beta$-catenin levels}

We next determined the expression of cytoplasmic and nuclear levels of the Wnt signaling intermediate $\beta$-catenin in CCA and CCC tissues. The immunohistochemistry images of $\beta$ catenin protein were shown in Figure 1, lower panel. The percentage of cases with cytoplasmic $\beta$-catenin (Figure 2C) was high in all three groups (HCCA: $98.5 \%$, IHCC: $77.4 \%$, and CCC: $75.6 \%)$. An adjusted alpha of 0.17 was utilized for multiple paired-comparisons between the groups. Levels of cytoplasmic $\beta$-catenin were significantly greater in HCCA than CCC samples $(\mathrm{P}<0.01)$, and greater in CCC samples than IHCC $(\mathrm{P}<0.01)$. The percentage of samples staining strongly positive for $\beta$-catenin was significantly higher in the HCCA group (81.9\%; Figure $2 \mathrm{C}++$ plus +++ ) than the IHCC group $(29.0 \%, \mathrm{P}<0.01)$, but not the CCC group (75.6\%, $\mathrm{P}=0.359)$. Nuclear levels of $\beta$ catenin were significantly greater in the HCCA group (66.9\%) than the IHCC group (41.9\%, $\mathrm{P}=0.012)$, but not the CCC group $(62.2 \%$, $\mathrm{P}=0.568$; Figure 2D).

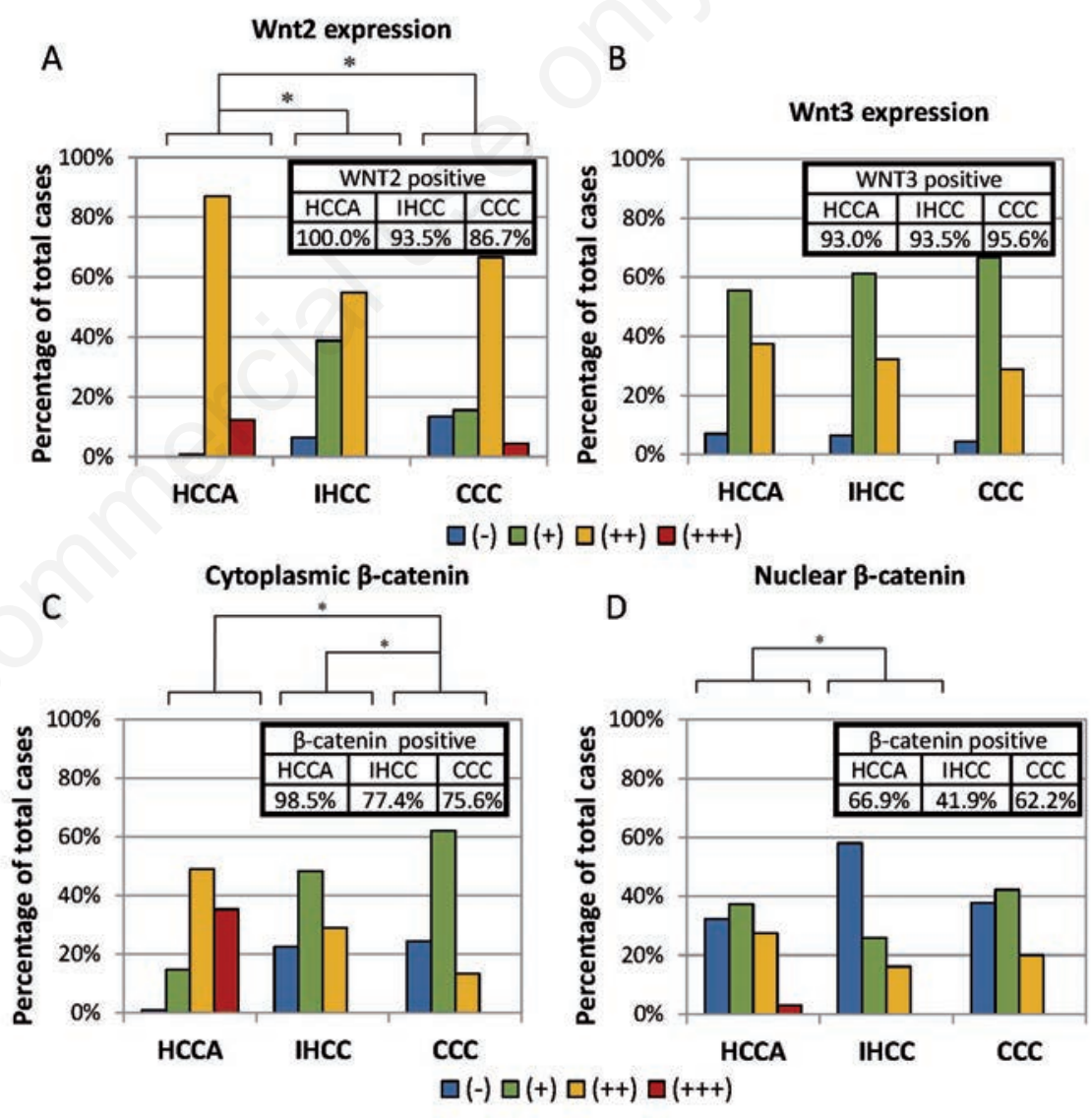

Figure 2. Production of Wnt2 (A) and Wnt3 (B) were quantified in tissues from HCCA, IHCC, or CCC patients. The percentage of the subjects from each group that stained positive or negative (blue bar) for Wnt is shown. Positive staining overall was defined as samples that were + (less than $10 \%$ of cells producing Wnt; green bar), ++ (10-49\% of cells producing Wnt; yellow bar), and +++ $(50 \%$ or more of the cells producing Wnt; red bar). Production of cytoplasmic $\beta$-catenin (C) and nuclear $\beta$-catenin (D) were quantified in tissues from HCCA, IHCC, or CCC patients. The percentage of the subjects from each group that stained positive or negative (blue bar) for $\beta$-catenin production is shown. Positive staining overall was defined as samples that were + (less than $10 \%$ of cells producing $\beta$-catenin; green bar $),++(10-49 \%$ of cells producing $\beta$-catenin; yellow bar $)$, and $+++(50 \%$ or more of the cells producing $\beta$-catenin; red bar $)$. Samples considered strongly positive were defined as ++ or +++ results. Statistically significant results $(P<0.05)$ are labeled with an asterisk. 


\section{Levels of TCF 4}

In the activated canonical Wnt pathway, $\beta$ catenin enters the nucleus, binds to TCF/LEF to form a complex, and activates downstream target genes. ${ }^{10}$ The immunohistochemistry images of TCF4 protein were shown in Figure 3 , upper panel. The percentage of patients expressing nuclear TCF4 was significantly higher in the HCCA group (86.5\%) than the IHCC $(64.5 \%, \mathrm{P}<0.001)$ and CCC groups (41.9\%, $\mathrm{P}<0.001$; Figure 4).

\section{Production of Wnt pathway target proteins c-Myc and cyclin D1}

To determine whether Wnt signaling was leading to activation of its downstream targets, the levels of cyclin D1 and c-Myc were evaluated. The immunohistochemistry images of cMyc protein were shown in Figure 3, middle panel. c-Myc expression was significantly higher in the HCCA samples (100\%) than the IHCC $(90.3 \%, \mathrm{P}<0.001)$ and CCC $(91.3 \%$, $\mathrm{P}<0.001$ ) samples (Figure 5A). The immunohistochemistry images of cyclin D1 protein were shown in Figure 3, lower panel. Cyclin D1 was also expressed at significantly higher levels in the HCCA group (98.4\%) than in the IHCC $(90.3 \%, \mathrm{P}<0.001)$ and CCC $(62.2 \%$, $\mathrm{P}<0.001$ ) groups (Figure $5 \mathrm{~B}$ ).

\section{Wnt pathway activation and partial} activation

Based on the protein levels of each marker, we classified the tissue samples as having Wnt pathway activation (Wnt2+, Wnt3+, nuclear $\beta$ catenin+, and nuclear TCF4+) or partial Wnt pathway activation (Wnt2+ or Wnt $3+$ and nuclear $\beta$-catenin+ or TCF4+). Of the HCCA samples, 45.7 had canonical Wnt activation (Figure 6A), which was significantly higher than the CCC group (29\%; $\mathrm{P}=0.003)$, but not the IHCC group (32.3\%; $\mathrm{P}=0.952)$. Similarly, the HCCA group had the highest percentage of samples with partial Wnt pathway activation (78\%), and was significantly greater than the IHCC group (61.3\%; $\mathrm{P}=0.005)$, but not the CCC group (73.3\%; $\mathrm{P}=0.115)$. Partial Wnt activation was not significantly different between the IHCC and CCC groups (Figure 6B).

\section{Correlation between Wnt pathway activation and HCCA stage}

The level of Wnt pathway activation did not correlate with histological staging using either the T-staging system (Figure 7A) or the Bismuch-Corlette classification index (Figure 7B).

Assessing the relationship between Wnt pathway factors and surgical resection outcomes

Based on the preoperative assessment,
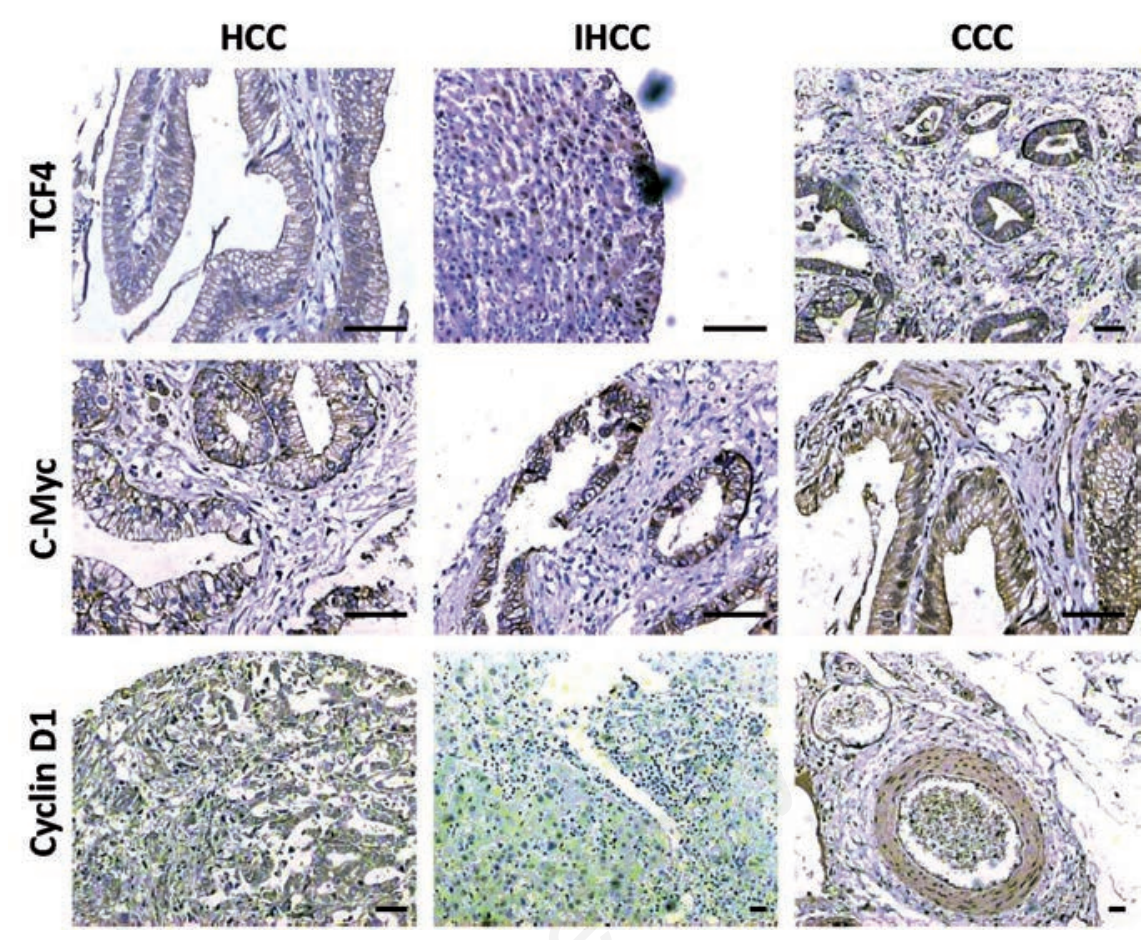

Figure 3. Representative immunohistochemistry images of TCF4, c-Myc and cyclin D1 proteins (upper, middle and lower panel, respectively). Scale bars: $100 \mu \mathrm{m}$.

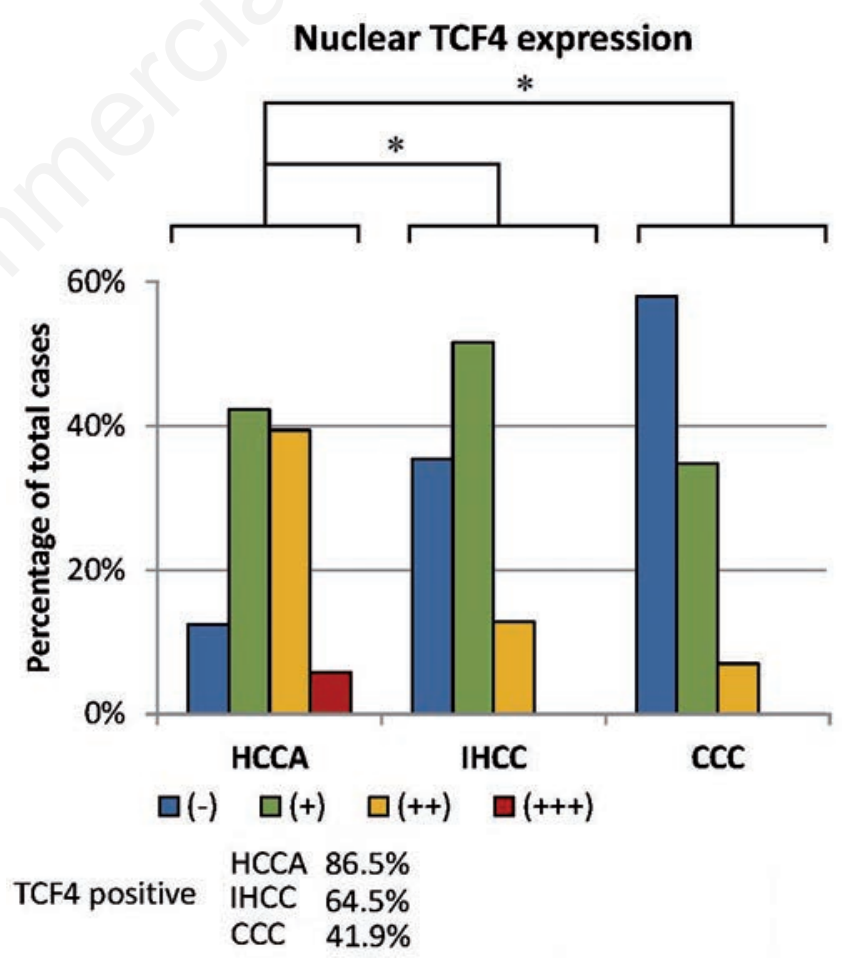

Figure 4. Production of nuclear TCF4 was quantified in tissues from HCCA, IHCC, or CCC patients. The percentage of the subjects from each group that stained positive or negative (blue bar) for nuclear TCF4 production is shown. Positive staining overall was defined as samples that were + (less than $10 \%$ of cells producing nuclear TCF4; green bar), ++ (10-49\% of cells producing nuclear TCF4; yellow bar), and $+++(50 \%$ or more of the cells producing nuclear TCF4; red bar). Samples considered strongly positive were defined as ++ or +++ results. Statistically significant results $(P<0.05)$ are labeled with an asterisk. 
patients who might be cured by tumor resection underwent surgery and were followed post-operatively. A multivariable analysis was applied to investigate if expression of any Wnt pathway proteins could be used as prognostic markers for surgical outcomes (complete resection, palliative resection, or exploratory laparotomy). The univariable analysis showed that cyclin D1 levels, Wnt pathway activation, and partial Wnt pathway activation were all significant predictors of surgical outcome (Table 1).

However, in multivariate logistic regression analysis, cyclin D1 was the only independent predictor of surgical outcome (estimate: $-1.763, \mathrm{P}=0.018$; odds ratio: $0.172,95 \%$ confidence interval: $-3.229,-0.298)$, suggesting cyclin D1 might be a prognostic marker for surgical resection outcomes.

\section{Relationship between Wnt path- way activation and the prognosis of HCCA patients}

Based on Wnt pathway activation HCCA patients were stratified into two groups and survival time was compared between the Wnt+ and Wnt- HCCA patients. The median survival period was 8 months for Wnt- patients and 11 months for Wnt+ patients (Figure 8). No significant differences in survival time were observed by a log-rank test, $\chi^{2}=0.045, \mathrm{P}=0.831$ or the Breslow test, $\chi^{2}=0.216, \mathrm{P}=0.642$.

\section{Determining the effect of Wnt pathway-related factors on prog- nosis}

A COX regression was used to determine risk factors affecting survival time and prognosis in HCCA patients (Table 2). Univariate analysis indicated that cyclin D1 levels and partial Wnt pathway activation were relevant risk factors. However, following multivariate COX regression analysis only partial Wnt pathway activation remained significantly associated with prognosis [ $(\mathrm{P}=0.018$; HR (hazard ratio): 3.152 ; HR (95\% confidence interval): $1.215,8.172]$.

\section{Discussion}

Wnt pathway activation has been linked to malignancy in many cancers and therefore might be useful as a prognostic indicator in patients with HCCA. Here, we used TMAs to assess Wnt activation in HCCA patients compared to patients with potentially malignant IHCC and relatively benign CCC. Wnt2 was present in all of the groups, but its levels were highest in the HCCA group and positively cor-
Table 1. Multivariable logistic regression analysis for factors affecting surgical outcome.

\begin{tabular}{|c|c|c|c|c|c|c|c|}
\hline Variate & Estimate & SE & Wald $\chi^{2}$ & df & $P$ & OR & $95 \%$ CI \\
\hline Cyclin D1 + & -1.763 & 0.748 & 5.560 & 1 & 0.018 & 0.172 & $-3.229 \sim-0.298$ \\
\hline
\end{tabular}

df, degree of freedom; OR, odds ratio; CI, confidence interval.

Table 2. Multivariable COX regression analysis for factors affecting prognosis.

\begin{tabular}{lcccccc} 
Variable & SE & Wald $\chi^{2}$ & if & P & HR & HR (95\% CI) \\
Partial Wnt pathway activation & 0.486 & 5.576 & 1 & 0.018 & 3.152 & $1.215 \sim 8.172$ \\
\hline
\end{tabular}

df, degree of freedom; HR, hazard ratio; CI, confidence interval.

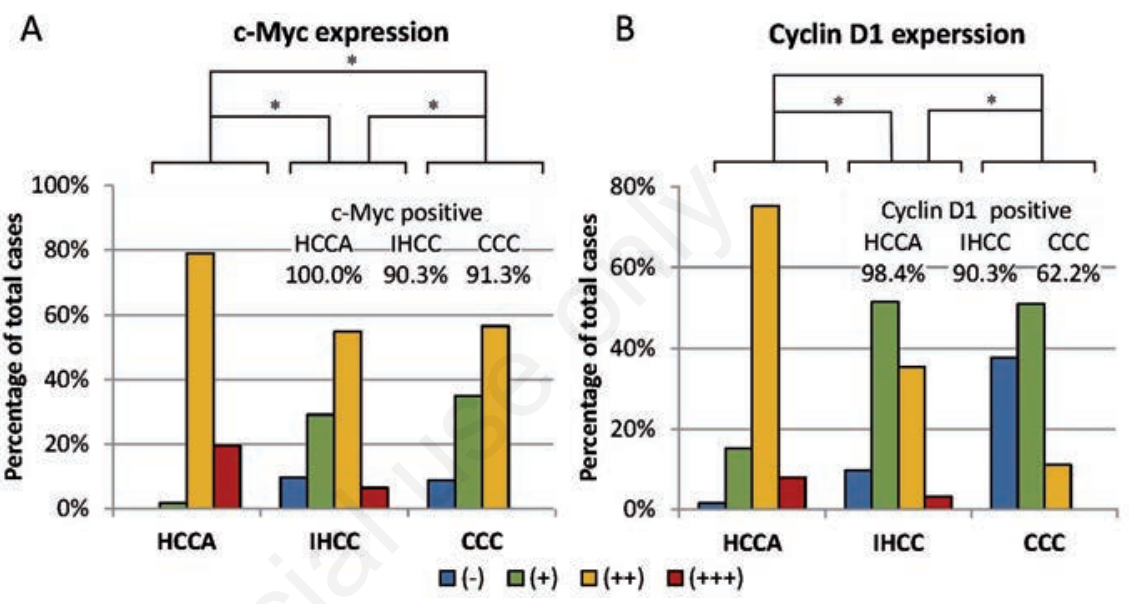

Figure 5. Production of c-Myc (A) and cyclin D1 (B) were quantified in tissues from HCCA, IHCC, or CCC patients. The percentage of the subjects from each group that stained positive or negative (blue bar) for protein production is shown. Positive staining overall was defined as samples that were + (less than $10 \%$ of cells positive; green bar),++ $(10-49 \%$ of cells positive; yellow bar $)$, and $+++(50 \%$ or more of the cells positive; red bar). Samples considered strongly positive were defined as ++ or +++ results. Statistically significant results $(P<0.05)$ are labeled with an asterisk.
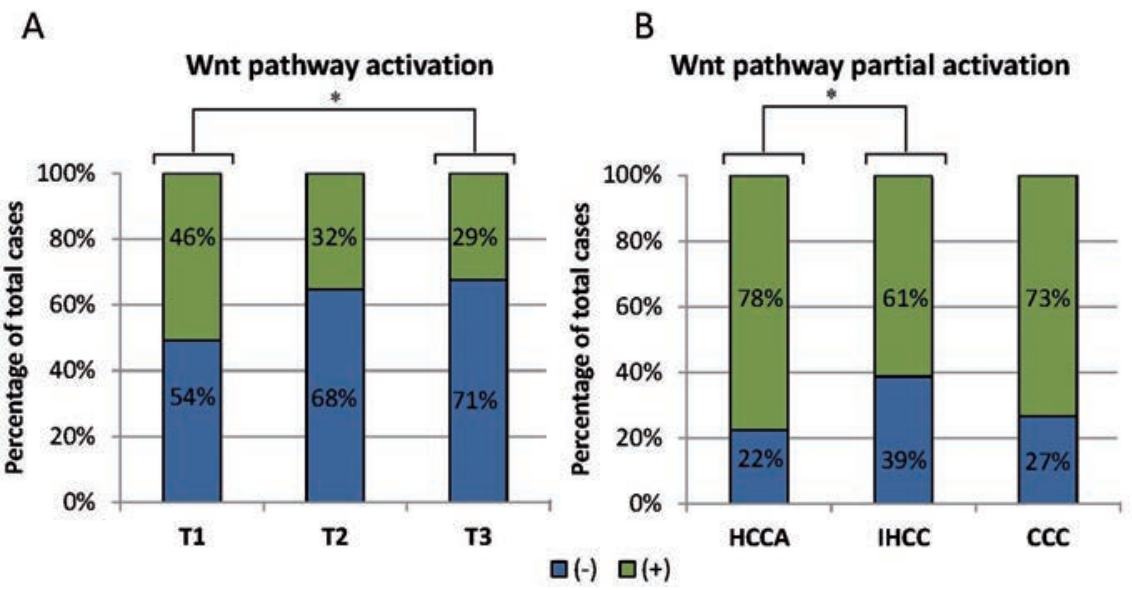

Figure 6. The overall activity of the Wnt signaling pathway was determined for each patient in the HCCA, IHCC, and CCC groups based on the production of Wnt2, Wnt 3 , $\beta$-catenin, and TCF4. Full Wnt pathway activation was defined as Wnt2+, Wnt3+, nuclear $\beta$-catenin+, and nuclear TCF4+ (A) and partial Wnt pathway activation was defined as Wnt $2+$ or Wnt $3+$ and nuclear $\beta$-catenin+ or TCF4+ (B). The percentage of patients that were positive (green) or negative (blue) in each group were compared. Statistically significant results $(P<0.05)$ are labeled with an asterisk. 
related with c-Myc expression. These findings suggest that during the development of HCCA, upregulation of Wnt2 might initiate the canonical Wnt signaling pathway, enhancing c-Myc production, and potentially leading to tumorigenesis. ${ }^{17}$

Wnt3 was present at lower levels than Wnt2, and was weakly expressed (+) in the HCCA, IHCC, and CCC samples. Wnt3 levels positively correlated with the presence of cytoplasmic $\beta$ catenin, suggesting that the Wnt3 pathway regulates cytoplasmic $\beta$-catenin production in HCCA. ${ }^{18}$ Although we observed accumulation of $\beta$-catenin in the cytoplasm, we were not able to address the underlying mechanism using our methodology. In hepatitis B virus (HBV)induced $\mathrm{HCC}^{19}$ and non-transformed hepatic cells ${ }^{20}$ Wnt3 is upregulated and interacts with Frizzled-7, which upregulates the $\beta$-catenin gene. However, additional experiments should be conducted to clarify whether the Wnt3/Frizzled7 interaction seen in hepatocellular carcinoma applies to HCCA as well.

The transcription factors $\beta$-catenin and TCF4 are major players in the Wnt pathway. In HCCA patients, the high levels of cytoplasmic $\beta$-catenin and its nuclear translocation suggest the canonical Wnt pathway might be aberrantly activated. Interestingly, in terms of $\beta$ catenin expression and localization, the HCCA group was comparable to the CCC group, not the IHCC group. The difference in outcome between HCCA and CCC suggests that an inhibitory mechanism, such as TCF4 might attenuate the Wnt pathway downstream in CCC. Nuclear TCF4 expression is also considered a marker and major effector of Wnt pathway activation. ${ }^{21,22}$ While TCF4 was abundant in the nucleus and cytoplasm of all three groups, protein levels were highest in the HCCA group followed by the IHCC group. The high levels of TCF4 in HCCA and IHCC suggested that nuclear transcription factors play a key role in canonical Wnt pathway in cholangiocarcinoma. In addition, the relatively low levels of TCF4 in CCC underscore its potentially important role in the development of HCCA. Understanding how Wnt is inhibited in CCC could be important to understanding the pathogenesis of HCCA and the contrasting outcomes of HCCA and CCC.

The downstream Wnt target genes c-Myc and cyclin D1 exhibited similar trends. We found that c-Myc was present at aberrantly high levels in $100 \%$ of HCCA tumor cells. In addition, most of the HCCA cells stained strongly positive and at levels higher than the IHCC and CCC groups. The level of c-Myc was also positively correlated to Wnt2 levels, suggesting that c-Myc might play an important role in the development of HCCA. Cyclin D1 was also expressed at aberrantly high levels in HCCA (98.4\%) compared to the IHCC and CCC groups. Cyclin D1 mediates the G1-S cell cycle transition and plays a significant role in many cancers. ${ }^{23,24}$ High expression of cyclin D1 was the only factor that independently predicted prognosis, suggesting that cyclin D1 could influence the genesis, development, and prognosis of HCCA. It is not clear whether cyclin D1 is only activated by the Wnt pathway in HCCA and further studies are required to clarify this point.
Overall, even though Wnt signaling was activated in all three cancers, the highest levels of activity were in the HCCA group. These results suggest an essential role for the Wnt pathway in tumorigenesis and proliferation in HCCA. Despite their common pathologic features, the differences in the level of Wnt pathway activation between the IHCC and HCCA groups implies that there may be different molecular mechanisms driving oncogenesis, such as
A

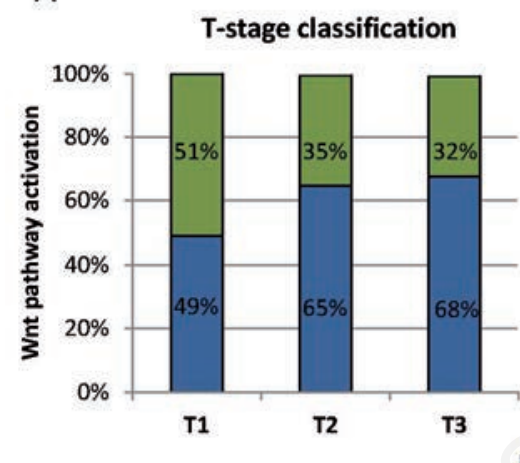

B

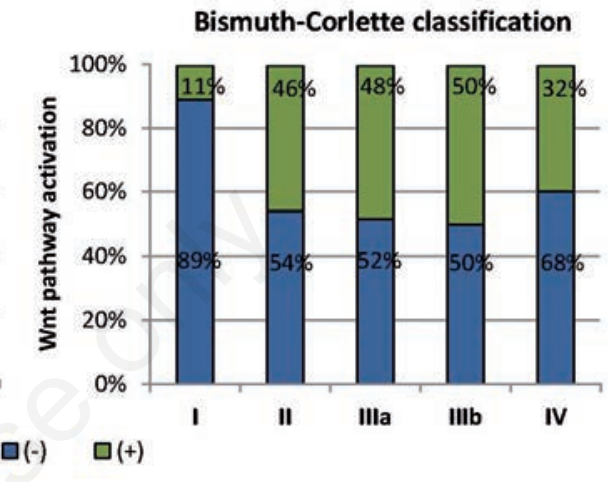

Figure 7. The HCCA patients were stratified based on overall activity of the Wnt signaling pathway and tumor staging using the T-stage system (A) or the Bismuth-Corlette system (B). Full Wnt pathway activation was defined as Wnt $2+$, Wnt3+, nuclear $\beta$-catenin+, and nuclear TCF4+ and partial Wnt pathway activation was defined as Wnt2+ or Wnt3+ and nuclear $\beta$-catenin + or TCF4+. The percentage of patients who were positive (green) or negative (blue) for Wnt signaling in each tumor stage were compared.

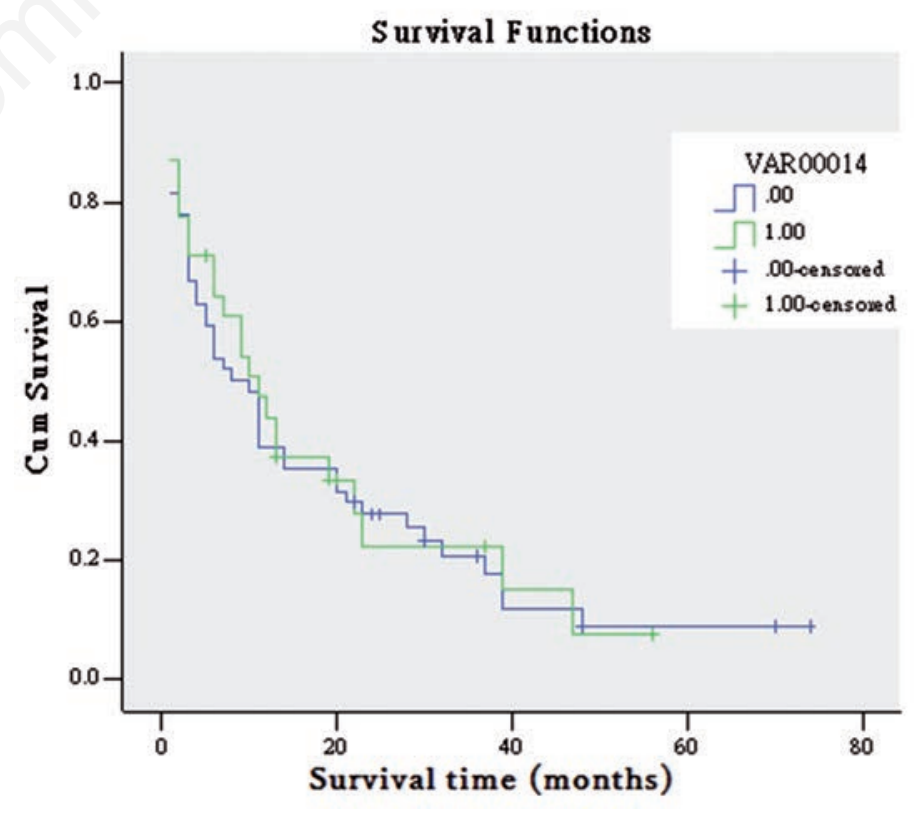

Figure 8. The HCCA patients were stratified based on overall activity of the Wnt signaling pathway and the survival time was compared between the patients that were positive (green) or negative (blue) for Wnt signaling. Full Wnt pathway activation was defined as Wnt2+, Wnt3+, nuclear $\beta$-catenin+, and nuclear TCF4+ and partial Wnt pathway activation was defined as Wnt2+ or Wnt3+ and nuclear $\beta$-catenin+ or TCF4+. The median survival time was not significantly different between groups. 
mutations of p53 and K-ras. ${ }^{16}$ The Wnt pathway was also activated in the CCC group but the activation level was much lower than in the HCCA and IHCC groups. We speculate that in CCC there may be inhibitory factors that block Wnt activation and prevent the malignant transforming effects of Wnt signaling. The role of the Wnt pathway in CCC requires further study because CCC belongs to a group of cancers characterized by inflammation, higher cell proliferation rates, and increased tumorigenesis. $^{25-27}$ Identifying a putative Wnt inhibitor that prevents malignancy in CCC may provide insights for treating HCCA.

A cancer staging system should ideally provide information about the prognosis and natural history of the disease. For cholangiocarcinoma the T-staging system and BismuthCorlette classification systems are the most widely applied staging systems. ${ }^{28}$ Wnt pathway activation did not correlate with tumor stage in either classification system, and survival time was not significantly different in HCCA patients stratified by Wnt activation (+/-). These results may be limited by the retrospective, cross sectional, nature of the study, and using protein levels to indicate pathway activation rather than a direct measure, such as luciferase assays to measure the expression of the Wnt and cyclin D1 encoding genes. However, the protein levels of cyclin D1, Wnt pathway activation, and partial Wnt pathway activation did contribute to determining whether a tumor could be resected and achieve $\mathrm{R}_{0}$ (microscopically negative tumor margins) results in univariable analyses. However, only cyclin D1 remained significantly associated with resection results in multivariable analyses. Therefore, although WNT pathway activation is not associated with prognosis, it does likely affect the outcome of surgical resection. In terms of prognosis, a multivariable COX analysis identified partial Wnt pathway activation as an independent predictor. The link between partial Wnt activation and prognosis indicated that Wnt signaling may play an important role in the occurrence of HCCA. The partial Wnt activation phenomenon may reflect inhibitory factors from other signaling pathways, or within the Wnt pathway, which prevent its full activation. The clinical significance and mechanisms underlying partial Wnt activation in HCCA warrant additional study.

In summary, using a multiplex histological analysis, we found high levels of proteins involved in canonical Wnt pathway activation and downstream target gene expression in HCCA. These results suggest that the Wnt signaling pathway may serve as a novel molecular mechanism governing the oncogenesis and development of HCCA.

\section{References}

1. Zabron A, Edwards RJ, Khan SA. The challenge of cholangiocarcinoma: dissecting the molecular mechanisms of an insidious cancer. Dis Model Mech 2013;6:281-92.

2. Hammill CW, Wong LL. Intrahepatic cholangiocarcinoma: a malignancy of increasing importance. J Am Coll Surg 2008;207:594-603.

3. Zaydfudim VM, Rosen CB, Nagorney DM. Hilar cholangiocarcinoma. Surg Oncol Clin N Am 2014;23:247-63.

4. Ruys AT, Busch OR, Rauws EA, Gouma DJ, van Gulik TM. Prognostic impact of preoperative imaging parameters on resectability of hilar cholangiocarcinoma. HPB Surg 2013;2013: 657309.

5. Saxena A, Chua TC, Chu FC, Morris DL. Improved outcomes after aggressive surgical resection of hilar cholangiocarcinoma: a critical analysis of recurrence and survival. Am J Surg 2011;202:310-20.

6. Suguru H, Iwao I, Hideaki F, Etsuro H, Yasuyuki S. Surgical resection of hilar cholangiocarcinoma: analysis of survival and postoperative complications. World J Surg 2007;31: 1256-63.

7. Jabło ska B. Biliary cysts: etiology, diagnosis and management. World $\mathrm{J}$ Gastroenterol 2012;18:4801-10.

8. Chen D, Zhu A, Zhang Z. Total laparoscopic Roux-en-Y cholangiojejunostomy for the treatment of biliary disease. JSLS 2013;17: 178-87.

9. Krausova M, Korinek V. Wnt signaling in adult intestinal stem cells and cancer. Cell Signal 2014;26:570-9.

10. Lustig B, Behrens J. The Wnt signaling pathway and its role in tumor development. $\mathrm{J}$ Cancer Res Clin Oncol 2003;129: 199-221.

11. Koehler A, Schlupf J, Schneider M, Kraft B, Winter C, Kashef J. Loss of Xenopuscadherin11 leads to increased Wnt/ $\beta$-catenin signaling and up-regulation of target genes c-myc and cyclin D1 in neural crest. Dev Biol 2013;383: 132-45.

12. Cowling VH, D’Cruz CM, Chodosh LA, Cole MD. c-Myc transforms human mammary epithelial cells through repression of the Wnt inhibitors DKK1 and SFRP1. Mol Cell Biol 2007;27:5135-46.

13. Zhang J, Han C, Wu T. MicroRNA-26a Promotes Cholangiocarcinoma Growth by Activating $\beta$-catenin. Gastroenterology 2012; 143:246-56.

14. Goeppert B, Konermann C, Schmidt CR, Bogatyrova 0, Geiselhart L, Ernst C, et al. Global alterations of DNA methylation in cholangiocarcinoma target the Wnt signaling pathway. Hepatology 2014;59:544-54.

15. Zhang KS, Zhou Q, Wang YF, Liang LJ. Inhibition of Wnt signaling induces cell apoptosis and suppresses cell proliferation in cholangiocarcinoma cells. Oncol Rep 2013;30:1430-8.

16. O'Dell MR, Huang JL, Whitney-Miller CL, Deshpande V, Rothberg P, Grose V, et al. Kras (G12D) and p53 mutation cause primary intrahepatic cholangiocarcinoma. Cancer Res 2012;72:1557-67.

17. Yoshida GJ, Saya H. Inversed relationship between CD44 variant and c-Myc due to oxidative stress-induced canonical Wnt activation. Biochem Biophys Res Commun 2014;443:622-7.

18. Wu Y, Ginther C, Kim J, Mosher N, Chung S,Slamon D, et al. Expression of Wnt3 activates $\mathrm{Wnt} / \beta$-catenin pathway and promotes EMT-like phenotype in trastuzumab-resistant HER2-overexpressing breast cancer cells. Mol Cancer Res 2012;10:1597-606.

19. Kim M, Lee HC, Tsedensodnom 0, Hartley R, Lim YS, Yu E, et al. Functional interaction between Wnt3 and Frizzled-7 leads to activation of the Wnt/beta-catenin signaling pathway in hepatocellular carcinoma cells. J Hepatol 2008;48:780-91.

20. Nambotin SB, Tomimaru Y, Merle P, Wands JR, Kim M. Functional consequences of WNT3/Frizzled7-mediated signaling in nontransformed hepatic cells. Oncogenesis 2012;1:e31.

21. Li X, Pu J, Jiang S, Su J, Kong L, Mao B, et al. Henryin, an ent-kauranediterpenoid, inhibits Wnt signaling through interference with $\beta$ catenin/TCF4 interaction in colorectal cancer cells. PLoS One 2013;8:e68525.

22. van Es JH, Haegebarth A, Kujala P, Itzkovitz S, Koo BK, Boj SF, et al. A critical role for the Wnt effectorTcf4 in adult intestinal homeostatic self-renewal. Mol Cell Biol 2012;32:1918-27.

23. Ramakrishna A, Shreedhar B, Narayan T, Mohanty L, Shenoy S, Jamadar S. Cyclin D1 an early biomarker in oral carcinogenesis. $\mathrm{J}$ Oral Maxillofac Pathol 2013;17:351-7.

24. Du B, Wang Z, Zhang X, Feng S, Wang G, He $\mathrm{J}$, et al. MicroRNA-545 suppresses cell proliferation by targeting Cyclin D1 and CDK4 in lung cancer cells. PLoS One 2014;9:e88022.

25. Aishima S, Kubo Y, Tanaka Y, Oda Y. Histological features of precancerous and early cancerous lesions of biliary tract carcinoma. J Hepatobiliary Pancreat Sci 2014;21: 448-52.

26. Ishibashi T, Kasahara K, Yasuda Y, Nagai H, Makino S, Kanazawa K. Malignant change in the biliary tract after excision of choledochal cyst. Br J Surg 1997;84:1687-91.

27. Ohashi T, Wakai T, Kubota M, Matsuda Y, Arai Y, Ohyama T, et al. Risk of subsequent biliary malignancy in patients undergoing cyst excision for congenital choledochal cysts. J Gastroenterol Hepatol 2013;28:243-7.

28. Suarez-Munoz MA, Fernandez-Aguilar JL, Sanchez-Perez B, Perez-Daga JA, GarciaAlbiach B, Pulido-Roa Y, et al. Risk factors and classifications of hilar cholangiocarcinoma. World J Gastrointest Oncol 2013;5:132-8. 